\title{
Searching for the biggest and the brightest; Selection of documents in VR-VIBE
}

\author{
Elizabeth F. Churchill, Dave Snowdon ${ }^{2}$, Steve Benford ${ }^{2}$ and Parmjit Dhanda ${ }^{2}$ \\ ${ }^{1}$ Dept. of Psychology, \\ University of Nottingham, \\ Nottingham NG7 2RD, UK \\ Elizabeth.Churchill@nottingham.ac.uk \\ ${ }^{2}$ Dept. of Computer Science, \\ University of Nottingham, \\ Nottingham NG7 2RD, UK \\ \{d.snowdon, s.benford\}@cs.nott.ac.uk
}

\section{INTRODUCTION}

This poster presents a preliminary usability evaluation of VR-VIBE (Benford et al., 1995). VR-VIBE is a 3-D information visualisation system which offers a relevance-based, visual representation of documents within a bibliographic database. Perceptual metaphors are exploited to direct the user's attention to salient items, whilst also providing meaningful information about the query-related salience of other items and about an item's 'relative relevance' to more than one topic.

\section{VR-VIBE}

VR-VIBE defines a technique for displaying document icons within a 3-D spatial framework of queries, called Points of Interest or POIs (Benford et al, 1995). The spatial position of an icon indicates the relative attraction of a document to the different queries or

\begin{abstract}
POIs, where attraction is estimated in terms of thematic similarity. Thus, an icon equally spaced between two POIs is equally relevant to both, while an icon close to a particular POI is relevant to that POI only. Absolute relevance is depicted by the size and brightness of the document's representation; the more relevant the document the bigger the icon and the brighter the colour. Relative icon size and brightness thus differentiates documents which are slightly relevant to all POIs from documents which are highly relevant to all POIs. Figure 1 illustrates a VR-VIBE display with 5 POIs (represented by green octahedrons) and 1582 documents (represent by blue cuboids). A 3D scrollbar allows users to filter out less relevant documents from the display by changing the relevance threshold. Users can navigate within the 3-D space, select individual documents, control the display according to the dynamic relevance threshold, "drag" POIs to new locations and specify new searches by creating new POIs or specifying keywords.
\end{abstract}




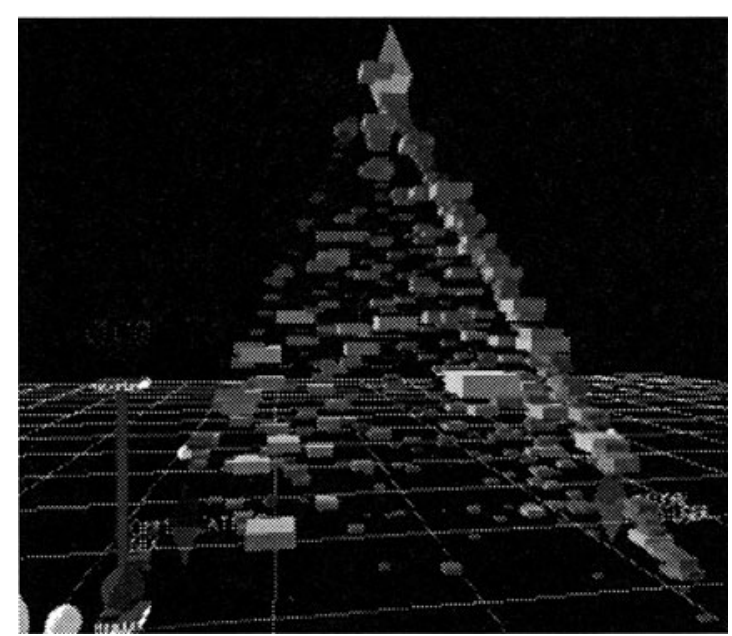

Figure 1: The world of documents in VR-VIBE.

\section{THE STUDY}

Although VR-VIBE has been designed to support multi-user, collaborative search and browsing, for the purposes of this preliminary usability study, we focused on individual users' search success and search strategies. Specifically, we considered: whether users are able to locate the most relevant document to a given query by exploiting the inter- and intra-icon cues, the methods used to specify searches and users' subjective assessments of the system.

15 graduate students from the University of Nottingham took part in the study. All participants were computer literate, although none had ever used 3$\mathrm{D}$ information visualisation tools before.

Seven study tasks were designed to test interface knowledge and ability to conduct semantically driven spatial analysis of the document space with only limited training. For each task, a target document was specified and users' selections compared to the target. The tasks ranged from highly specified to less specified. In later tasks, participants were given more freedom to select their own keywords for a search. Participants read two short introductory paragraphs on VR-VIBE and completed a number of practice tasks. The maximum time for practice was 40 minutes, after which participants completed the study tasks. Participants were video-taped throughout the sessions. Finally, a post-task questionnaire was presented and an interview conducted to establish participants' subjective judgements of and conceptual knowledge about the system. Item selections were evaluated in terms of time to make a selection and accuracy of that selection, determined by considering the overall relevance rating assigned to the selected document by VR-VIBE. Subjective measures were attained through presentation of a questionnaire.

\section{CONCLUSIONS}

This preliminary evaluation of VR-VIBE suggests that novice users with little training are able to select documents relevant to several POIs using spatial position and item features (size and brightness) as cues. Overall, less time for selection and better accuracy was achieved on the tasks with more POIs, but less document icons displayed. Analysis of the video tapes suggested that participants who scored the highest accuracy scores frequently used POI drags, exploiting the dynamic nature of the representation to disambiguate the spatial location of documents and thus their relative and absolute relevance. Tasks which required the specification of keywords resulted in the lowest accuracy measures. This suggests that whilst browsing an existing set of documents is facilitated by the visual display, specifying the keywords for the search presents more difficulties. This problem is not unique to search using VR-VIBE; selecting appropriate keywords often presents difficulties when using text-based systems (Borgman, 1986).

Although the results are promising, our study does not address the effect of practice on search strategy and success. Studies are currently being conducted to address this issue and to compare VR-VIBE with textually based search tools.

\section{REFERENCES}

Benford, S. Snowdon, D. Greenhalgh, C., Ingram, R., Know, I. and Brown, C. (1995) VR-VIBE A Virtual Environment for Co-Operative Information Retrieval, Proceedings of EuroGraphics '95, Maastricht, Holland.

Borgman, C.L. (1986) Why are online catalogs hard to use? Lessons learned from information retrieval studies. Journal of the American Society for Information Science, 37(6), 387-400. 